\title{
The Role of Transcranial Grayscale and Doppler Ultrasound Examination in Diagnosis of Neonatal Hypoxic-ischemic Encephalopathy
}

Yasmin H. Hemeda*, Zeinab A. Aly, Adel M. Al-Ghannam

Department of Radiodiagnosis, Faculty of Medicine, Menoufia University, Menoufia, Egypt

*Corresponding Author: Yasmin H. Hemeda, Mobile: (+20)01061173115, E-mail: hosneyyasmin@ gmail.com

\begin{abstract}
Background: The role of transcranial grayscale ultrasound (TC-GSUS) and transcranial color Doppler (TCD) in the diagnosis and prognosis of neonatal hypoxic-ischemic encephalopathy (HIE) is still questionable.

Objective: This study targeted to evaluate the role of TC-GSUS and TCD in diagnosis and prediction of the outcome of neonates with suspected HIE in comparison to Sarnat's clinical scoring.

Patients and methods: 26 neonates with suspected HIE were clinically evaluated and the severity of HIE was categorized according to Sarnat's clinical staging. Then, all neonates underwent sonographic examinations. TC-GSUS was performed at levels of anterior, mastoid, and posterior fontanelles and the level of the temporal window.

Results: Cranial biometry had negative and positive rates for HIE of $7.7 \%$ and $92.3 \%$, respectively. Using TC-GSUS, periventricular leukomalacia, intraventricular hemorrhage, brain edema, and hydrocephalus were detected in 17, 19, 14, and 16 patients, respectively. According to the resistive index (RI) of intracranial vessels, TCD excluded HIE in 11 patients and assured diagnosis of HIE with varying severity in 15 patients. Five neonates died and four developed neurological affection during follow-up. The outcome was correlated with Sarnat's scoring, ventricular-hemispheric ratio, and abnormalities of RI. Statistical analyses defined severity of HIE as judged by RI as the significant predictor for mortality and abnormal RI of anterior cerebral (ACA) and internal carotid arteries (ICA) are the most significant predictors of outcomes.

Conclusion: TCD can diagnose HIE in neonates with high sensitivity and specificity and abnormal RI of ICA and ACA might be used as valuable diagnostic and prognostic tests.

Keywords: Neonatal hypoxic-ischemic encephalopathy, Transcranial Doppler, Gray-scale ultrasound, Resistive index, Sarnat's clinical staging.
\end{abstract}

\section{INTRODUCTION}

Hypoxic-ischemic encephalopathy (HIE) is prevailing leading to severe brain damage affecting neonates ${ }^{[1]}$. HIE mostly affects preterm neonates, but also may affect term neonates and obstetric complications especially placental abruption, which had a strong association with HIE. Previous section, ruptured uterus, and shoulder dystocia are often associated with a high prevalence of HIE ${ }^{[2]}$. Perinatal asphyxia and/or cerebral ischemia and reperfusion can cause brain injury, which is often associated with subsequent neurological deficits, such as cerebral palsy or mental retardation ${ }^{[3]}$.

Moreover, infants with HIE had poorer motor repertoire, in comparison with their peers, and were seriously affected by HIE severity ${ }^{[4]}$. Clinically, HIE most commonly present by neonatal seizures, which are the most common neurological event in newborns that show higher prevalence in preterm than in full-term infants ${ }^{[4]}$. Sensorineural hearing loss ${ }^{[5]}$ or cerebral palsy with subsequent learning disabilities ${ }^{[6]}$ are other clinical sequelae of HIE. Early diagnosis of the extent and severity of neonatal brain injury secondary to HIE is an important issue because of the serious sequelae of HIE, but it remains a dilemma ${ }^{[7]}$.

MRI and DWI imaging has high detection rates for the diagnosis of hypoxic-ischemic encephalopathy [8]. Perfusion MRI scan can accurately reflect neonatal cerebral blood flow ${ }^{[9]}$, but its effect is limited due to the use of a gadolinium contrast agent, lack of replicated testing secondary to a cumulative effect, and other factors ${ }^{[10]}$.

MRI and MR spectroscopy provides early biomarkers of brain injury and treatment response in neonates with HIE, but its applicability is challenging. The 3D pseudo-continuous arterial spin-labeled technique provides several advantages, as the nonuse of a contrast agent or radiation and repeatable testing, over perfusion MRI technique [11]. However, postmortem examinations frequently show cerebellar injury in infants with severe HIE, while it is less well visible on MRI ${ }^{[12]}$.

Transcranial sonography (TCS) is a widely used non-invasive bedside method to evaluate the brain, its vessels, perfusion, and pathologies ${ }^{[12]}$.

Transcranial Doppler ultrasonography (TCD) is a real-time physiologic monitor that can detect altered cerebral hemodynamics during catastrophic brain injury ${ }^{[13]}$, allows both imaging of parenchymal structures and Doppler assessment of intracranial vessels ${ }^{[14]}$.

TCDS is a generally safe, repeatable, noninvasive technique that has a strong potential in neurocritical care patients in many clinical scenarios and is applicable for pediatric patients to allow for better care of children with neurologic insults ${ }^{[15]}$. This study targeted to evaluate the role of transcranial grayscale ultrasound (TC-GSUS) and transcranial color Doppler 
(TCD) in diagnosis and prediction of the outcome of neonates with suspected HIE in comparison to Sarnat's clinical scoring.

\section{PATIENTS AND METHODS}

The study was conducted through the period from May 2019 till June 2020. All neonates aged 1-28 days and have suspected diagnosis of HIE were evaluated for eligibility to be included in the study.

Collected clinical data included maternal data regarding parity, gestational duration, and mode of delivery. Neonatal data including 5-min Apgar score, need for resuscitation, mechanical ventilation and age at the time of inclusion. Clinical examination at the time of inclusion was performed by the neonatologist in charge, but the radiologist was blinded about the diagnosis and the obtained clinical data.

Neonates were clinically evaluated for items of Sarnat's score, which includes the level of consciousness, activity, neuromuscular control including determination of muscle tone, posture and stretch reflexes, primitive reflexes including suckling reflex, Moro reflex, and presence of tonic neck and for autonomic functions as heart rate and size of the pupils and presence of seizures ${ }^{[16]}$. Neonates were staged according to Sarnat's score into Stage I (mild HIE), Stage II (Moderate HIE), and Stage III (Severe HIE).

Exclusion criteria: The only exclusion criterion was neonatal oxygen saturation of $<90 \%$, which necessitates special transportation conditions.

Inclusion criteria: Neonates with a suspected diagnosis of HIE and who could be transported to the Diagnostic Radiology Department were included in the study.

\section{Image acquisition:}

Twenty-six sonographic examinations were performed using SonoScape SSI-6000 ultrasound device that is equipped by L743 linear array probe with frequency 5-10 $\mathrm{MHz}$. Transcranial examination at levels of anterior, mastoid, and posterior fontanelles and at the level of the temporal window was conducted as previously described by Meijler and Steggerda ${ }^{[17]}$. Color-coded Doppler study of the intracerebral vessels was performed according to Meijler and Steggerda ${ }^{[18]}$ to assess brain perfusion and to detect ischemic brain changes.

\section{Procedure:}

Neonates were kept in a quiet state in the supine position. Firstly, through the anterior fontanelle and on sagittal and coronal planes morphological structures of brain tissue. Size and location of US abnormalities and size and shape of lateral ventricles (Lat-V) were assessed. Also, TCD was used to assess the blood flow parameters of the anterior cerebral artery (ACA). Then, through temporal cross-section, to display skull base arterial rings, blood flow parameters of the middle cerebral artery (MCA) were determined. The determined blood flow parameters included systolic velocity (Vs) and diastolic velocity (Vd), and resistance index (RI) was calculated as follows: $\mathrm{RI}=(\mathrm{Vs}-\mathrm{Vd}) / \mathrm{Vs}$ [19].

RI was differentiated as normal or abnormal according to gestational age and abnormal RI in preterm neonates, which is considered abnormal if it was $<0.54$ or $>0.86$ for preterm neonates and if it was $<0.52$ or $>$ 0.8 for term neonates ${ }^{[20]}$.

\section{Study outcome:}

1. Primary outcome: is the diagnostic validity of TC-GSUS versus TCD as diagnostic procedures for neonatal HIE

2. Secondary outcome: is the prognostic validity of TC-GSUS and TCD, and Sarnat's clinical staging system for prediction of outcome defined as mortality and development of neurological deficit as documented by the neonatologist in charge.

\section{Sample size:}

Previous studies for diagnosis and stratification of cases with suspected neonatal HIE included $20^{[21]}$ and 21 patients ${ }^{[22]}$ to approach significant differences. The current study supposed a $40 \%$ difference between positive predictive value (PPV) of TC-GSUS and TCD would reach significant difference with a study power of $80 \%, \alpha$ value of 0.05 , and $\beta$ value of 0.2 on evaluation of a minimum of 23 patients per group.

\section{Ethical consent:}

An approval of the study was obtained from Menoufia University Academic and Ethical Committee. Every patient signed an informed written consent for acceptance of the study. This work has been carried out in accordance with The Code of Ethics of the World Medical Association (Declaration of Helsinki) for studies involving humans.

\section{Statistical analysis}

Obtained data were presented as mean, standard deviation, numbers, and percentages. Sensitivity \& specificity of studied parameters as predictors were evaluated using the receiver operating characteristic (ROC) curve analysis judged by the area under the curve (AUC) compared versus the null hypothesis that $\mathrm{AUC}=0.05$ and paired-sample AUC differences were determined to the null hypothesis that $\mathrm{AUC}=0$. Regression analysis (Stepwise method) was used for the stratification of studied parameters as specific predictors. Statistical analysis was conducted using the SPSS (Version 15, 2006) for Windows statistical package. P-value $\leq 0.05$ was considered statistically significant. 


\section{RESULTS}

The study included 26 neonates with suspected HIE with a mean age at the time of presentation of $6 \pm 3.9$; ranging from 2-16 days and mean weight registered at the birth of $3232 \pm 619$; ranging from 2150-4000 gm. The 5-min Apgar score was $\leq 5$ in 3 patients, 5 patients required resuscitation, 4 required mechanical ventilation, and $7 \mathrm{had}$ at least one attack of symptomatic seizures. Clinically, 19 patients were Sarnat's score of I, 5 patients were Sarnat's score II and two patients were Sarnat's score 3. Unfortunately, during the hospital stay, 5 patients died and four survivors developed neurological handicapping during follow-up as shown in table (1) and figure (1).

Table (1): Patients' clinical data

\begin{tabular}{|c|c|c|c|c|}
\hline \multicolumn{4}{|l|}{ Data } & Findings \\
\hline \multirow{7}{*}{$\begin{array}{l}\text { Maternal } \\
\text { data }\end{array}$} & \multirow{2}{*}{\multicolumn{2}{|c|}{ Maternal parity }} & Nullipara & $14(53.9 \%)$ \\
\hline & & & Multipara & $12(46.1 \%)$ \\
\hline & \multirow{2}{*}{\multicolumn{2}{|c|}{ Gestational duration }} & Full-term & $16(61.5 \%)$ \\
\hline & & & Preterm & $10(38.5 \%)$ \\
\hline & \multirow{3}{*}{\multicolumn{2}{|c|}{ Mode of delivery }} & Normal vaginal & $5(19.2 \%)$ \\
\hline & & & Difficult vaginal & $7(26.9 \%)$ \\
\hline & & & Cesarean section & $14(53.9 \%)$ \\
\hline \multirow{20}{*}{$\begin{array}{l}\text { Neonatal } \\
\text { data }\end{array}$} & \multirow{2}{*}{\multicolumn{2}{|c|}{ APGAR score at 5-min }} & $0-5$ & $3(11.5 \%)$ \\
\hline & & & $6-10$ & $23(88.5 \%)$ \\
\hline & \multirow{2}{*}{\multicolumn{2}{|c|}{ Gender }} & Males & $15(57.7 \%)$ \\
\hline & & & Females & $11(42.3 \%)$ \\
\hline & \multirow{2}{*}{\multicolumn{2}{|c|}{ Neonatal resuscitation }} & Yes & $5(19.2 \%)$ \\
\hline & & & No & $21(80.8 \%)$ \\
\hline & \multirow{2}{*}{\multicolumn{2}{|c|}{ Mechanical ventilation }} & Yes & $4(15.4 \%)$ \\
\hline & & & No & $22(84.6 \%)$ \\
\hline & \multirow{2}{*}{\multicolumn{2}{|c|}{ Symptomatic seizures }} & Yes & $7(26.9 \%)$ \\
\hline & & & No & $19(73.1 \%)$ \\
\hline & \multirow{4}{*}{\multicolumn{2}{|c|}{ Birth weight (gm.) }} & $<2500$ & $5(19.2 \%)$ \\
\hline & & & $2500-3000$ & $3(11.5 \%)$ \\
\hline & & & $>3000$ & $18(69.3 \%)$ \\
\hline & & & Mean \pm SD & $3232 \pm 619(2150-4000)$ \\
\hline & \multirow{3}{*}{\multicolumn{2}{|c|}{$\begin{array}{l}\text { Neonatal age at the time of } \\
\text { enrolment (days) }\end{array}$}} & $\leq 7$ & $16(61.5 \%)$ \\
\hline & & & $>7$ & $10(38.5 \%)$ \\
\hline & & & Mean \pm SD & $6 \pm 3.9(2-16)$ \\
\hline & \multirow{3}{*}{\multicolumn{2}{|c|}{ Sarnat's clinical scoring }} & Stage I & $19(73.1 \%)$ \\
\hline & & & Stage II & $5(19.2 \%)$ \\
\hline & & & Stage III & $2(7.7 \%)$ \\
\hline \multirow{4}{*}{\multicolumn{2}{|c|}{ Neonatal outcome }} & \multirow{2}{*}{ Mortality } & Died & $5(19.2 \%)$ \\
\hline & & & Survived & $21(80.8 \%)$ \\
\hline & & \multirow{2}{*}{ Neurologic outcome } & Normal & $17(81 \%)$ \\
\hline & & & Handicapped & $4(19 \%)$ \\
\hline
\end{tabular}

Data are presented as mean, standard deviation (SD), range, numbers, and percentages 


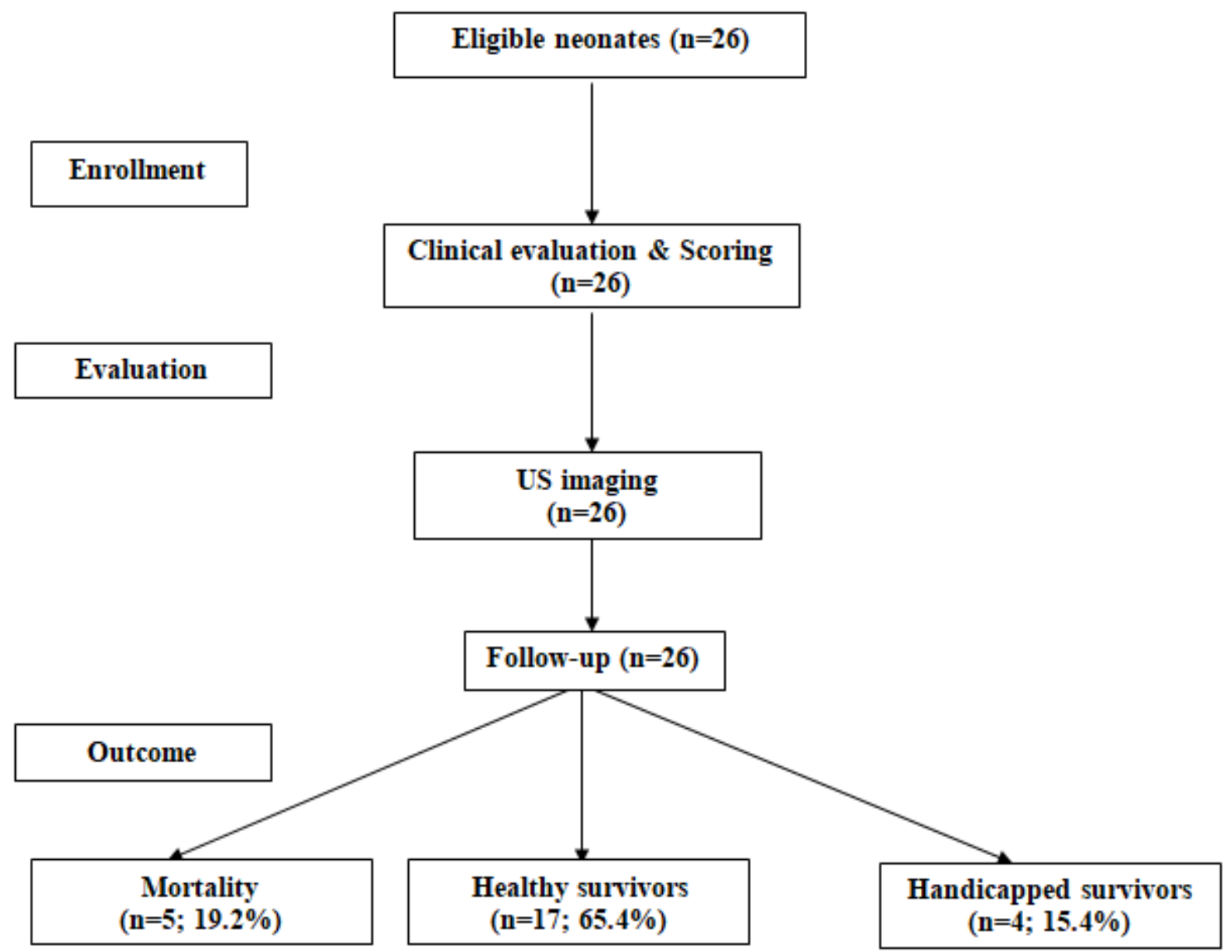

Fig. (1): Study Flow Chart

\section{Gray-scale US findings}

Gray-scale cranial biometry findings were normal in only two patients $(7.7 \%)$, while 24 patients had at least an abnormal finding (Table 2). According to cranial biometry findings, TC-GSUS has negative and positive rates for neonatal HIE of $7.7 \%$ and $92.3 \%$, respectively. Using TC-GSUS, periventricular leukomalacia was detected in 17 patients (65.4\%), intraventricular hemorrhage in 19 patients (73.1\%), and brain edema and hydrocephalus in $14(53.8 \%)$ and 16 patients $(61.5 \%)$, respectively (Table 3$)$.

Table (2): Distribution of studied patients according to results of Gray-scale cranial biometry

\begin{tabular}{|c|c|c|c|}
\hline Signs & \multicolumn{2}{|c|}{ Frequency } & Mean ( \pm SD) \\
\hline \multirow[t]{2}{*}{ Sino-cortical width (mm) } & $\mathrm{NCP}(<3)$ & $22(84.6 \%)$ & \multirow{2}{*}{$1.49( \pm 0.96)$} \\
\hline & Out of NCP & $4(15.4 \%)$ & \\
\hline \multirow{2}{*}{ Cranio-cortical width (mm) } & $\mathrm{NCP}(<4)$ & $20(76.9 \%)$ & \multirow{2}{*}{$2.404( \pm 1.76)$} \\
\hline & Out of NCP & $6(23.1 \%)$ & \\
\hline \multirow[t]{2}{*}{ Inter-Hemispheric width (mm) } & $\mathrm{NCP}(<5)$ & $22(84.6 \%)$ & \multirow{2}{*}{$2.235( \pm 1.57)$} \\
\hline & Out of NCP & $4(15.4 \%)$ & \\
\hline \multirow[t]{2}{*}{ Lateral ventricle width $(\mathrm{mm})$} & $\mathrm{NCP}(<13)$ & $12(46.1 \%)$ & \multirow{2}{*}{$14.05( \pm 5.9)$} \\
\hline & Out of NCP & $14(53.9 \%)$ & \\
\hline \multirow[t]{2}{*}{$3^{\text {rd }}$ ventricle width $(\mathrm{mm})$} & $\mathrm{NCP}(<3)$ & $12(46.1 \%)$ & \multirow{2}{*}{$6.57( \pm 5.46)$} \\
\hline & Out of NCP & $14(53.9 \%)$ & \\
\hline \multirow{3}{*}{ Hemispheric width (mm) } & $<\mathrm{NCP}(<20)$ & 0 & \multirow{3}{*}{$34.7( \pm 6.5)$} \\
\hline & Within normal range (20-45) & $22(84.6 \%)$ & \\
\hline & $>\mathrm{NCP}(>45)$ & $4(15.4 \%)$ & \\
\hline \multirow[t]{3}{*}{ Ventricular-hemispheric Ratio } & $<\mathrm{NCP}(<20)$ & $3(11.5 \%)$ & \multirow{3}{*}{$41.65( \pm 18.8)$} \\
\hline & Within normal range (20-34) & $8(30.9 \%)$ & \\
\hline & $>\mathrm{NCP}(>34)$ & $15(57.6 \%)$ & \\
\hline
\end{tabular}

Data are presented as mean, standard deviation (SD), numbers, percentages; $\quad$ NCP: Normal cutoff point 
https://ejhm.journals.ekb.eg/

Table (3): Distribution of studied patients according to Gray-scale findings

\begin{tabular}{|l|l|c|}
\hline \multirow{4}{*}{$\begin{array}{l}\text { Periventricular } \\
\text { leukomalacia }\end{array}$} & Negative & Findings \\
\cline { 2 - 3 } & Grade1 & $9(34.6 \%)$ \\
\cline { 2 - 3 } & Grade 2 & $7(23.1 \%)$ \\
\cline { 2 - 3 } & Grade 3 & $3(11.6 \%)$ \\
\cline { 2 - 3 } Intraventricular & Grade 4 & $1(3.8 \%)$ \\
\hline \multirow{5}{*}{ hemorrhage } & Negative & $7(26.9 \%)$ \\
\cline { 2 - 3 } & Grade1 & $6(23.1 \%)$ \\
\cline { 2 - 3 } & Grade 2 & $6(23.1 \%)$ \\
\cline { 2 - 3 } Brain Edema & Grade 3 & $7(26.9 \%)$ \\
\hline \multirow{3}{*}{ Hydrocephalus } & Negative & $14(53.9 \%)$ \\
\cline { 2 - 3 } & Positive & $12(46.1 \%)$ \\
\cline { 2 - 3 } & Negative & $16(61.5 \%)$ \\
\cline { 2 - 3 } & Positive & $10(38.5 \%)$ \\
\hline
\end{tabular}

Data are presented as numbers, percentages

Doppler studies detected abnormal RI of ACA, MCA and ICA in 11, 12 and, 13 patients, respectively (Table 4). According to disturbed RI of intracranial vessels, Doppler scanning excluded the presence of HIE in 11 patients (42.3\%) and assured the diagnosis of HIE in 15 patients (57.7\%); 3 patients (11.5\%) had grade 4, 8 patients $(30.8 \%)$ had grade 3 , and 4 patients (15.4\%) had grade 2 HIE.

Table (4): Distribution of studied patients according to RI of cranial arteries as judged by transcranial US Doppler

\begin{tabular}{|l|c|c|c|}
\hline \multirow{2}{*}{ Anterior cerebral artery RI } & \multicolumn{2}{|c|}{ Frequency of } & Median [IQR] \\
\cline { 2 - 3 } & Normal & $11(42.3 \%)$ & \multirow{2}{*}{0.64 [0.54-0.93] } \\
\cline { 2 - 3 } & Abnormal & $15(57.7 \%)$ & \\
\cline { 1 - 3 } Middle cerebral artery RI & Normal & $12(46.1 \%)$ & \multirow{2}{*}{0.67 [0.55-0.92] } \\
\cline { 2 - 3 } & Abnormal & $14(53.9 \%)$ & \multirow{2}{*}{$0.695[0.57-0.94]$} \\
\cline { 2 - 3 } & Normal & $13(50 \%)$ & \\
\cline { 2 - 3 } & Abnormal & $13(50 \%)$ & \\
\hline
\end{tabular}

RI: Resistive index; IQR: Interquartile range 


\section{Case Models \\ CASE (1)}

A newly born 3 days old preterm male baby was delivered after a difficult normal vaginal delivery for a nulliparous woman and presented by repeated attacks of convulsions on the ventilator. Images in coronal planes showed dilatation of both lateral ventricles ( $16 \& 19 \mathrm{~mm}$ ) with left-sided intraventricular hemorrhage (IVH) and blood clot formation, dilatation of $3^{\text {rd }}$ ventricle $(14 \mathrm{~mm})$, increased lateral ventricle/hemispheric ratio. Images in axial planes showed dilatation of the $3^{\text {rd }}$ ventricle $(11 \mathrm{~mm})$ and cystic dilatation of the $4^{\text {th }}$ ventricle. Images in sagittal planes showed dilatation of lateral ventricle with hemorrhagic choroid plexus as well as IVH and blood clot formation, cystic degeneration of periventricular white matter denoting periventricular leukomalacia (PVL) grade 3. Doppler study of ICA and ACA showed increased resistive index ( $\mathrm{RI}=0.97 \& 0.91$, respectively) with bare loss of diastolic component. According to wave shape and spectral analysis, this case had grade 4 hypoxic-ischemic brain injury (Fig. 2).
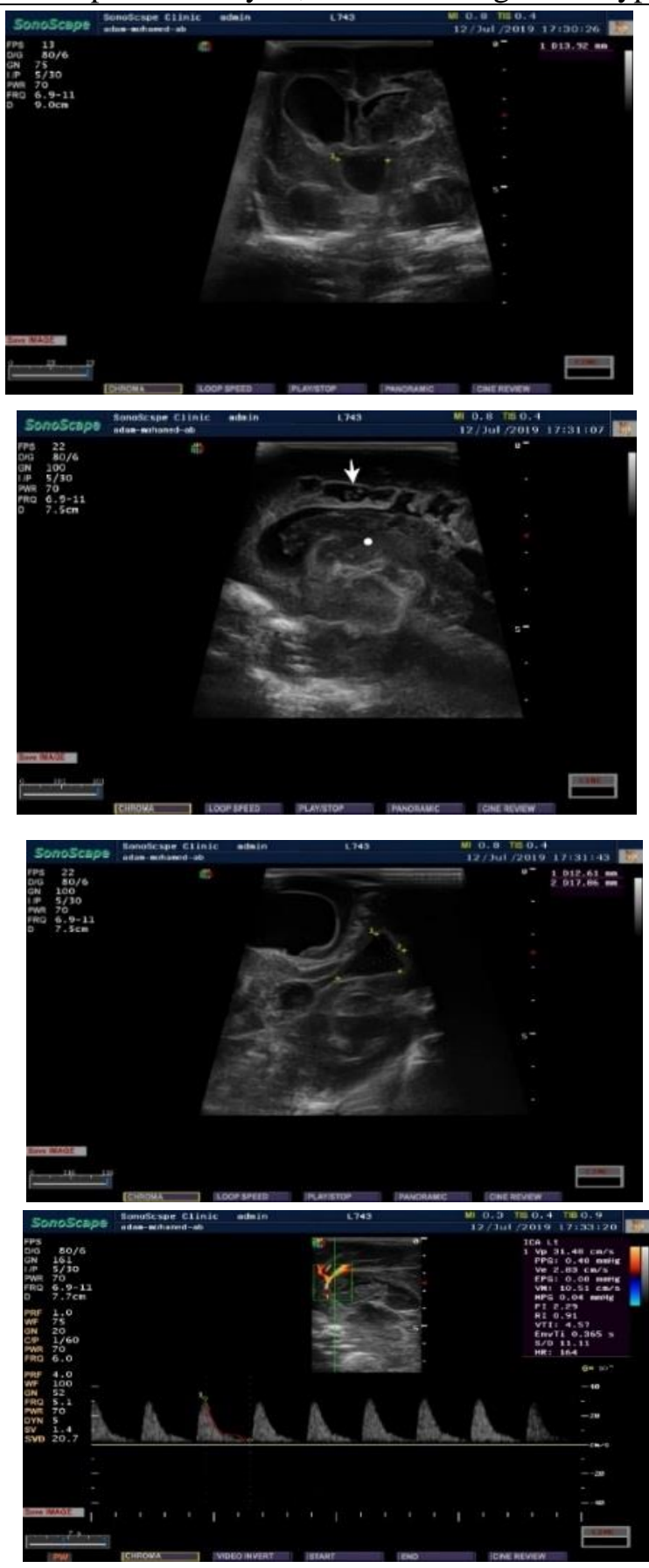
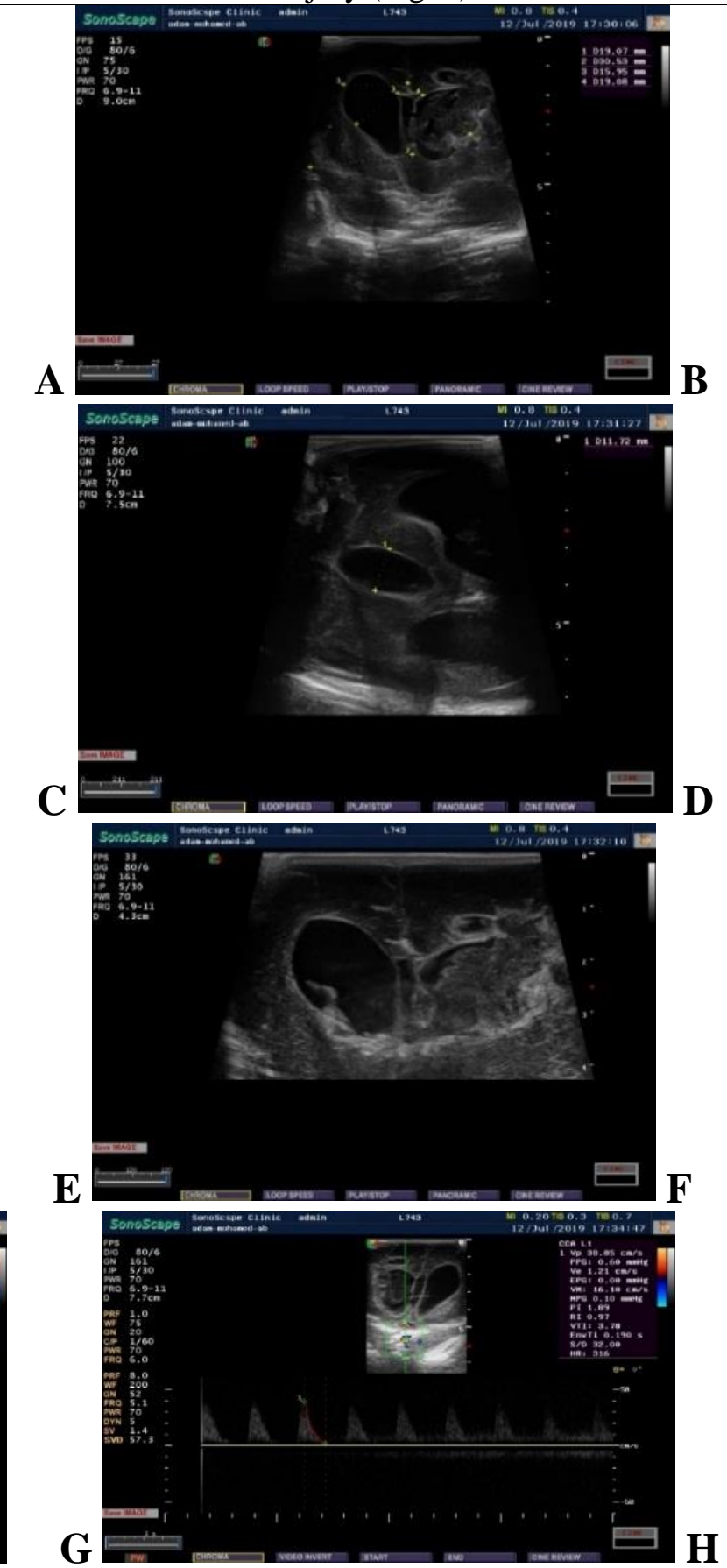

Fig. (2): Coronal plane US showed dilatation of the $3^{\text {rd }}$ and lateral ventricles with left sided IVH and blood clot formation as well as increased lateral ventricle/hemispheric ratio (a \& b). Sagittal plane US showed dilated lateral ventricle with hemorrhagic choroid plexus as well as IVH and blood clot formation (dot) with cystic degeneration of periventricular white matter denoting PVL grade 3 (c \& d). Axial plane US showed dilatation of the $3^{\text {rd }}$ ventricle and cystic dilatation of the $4^{\text {th }}$ ventricle (e \& f). Doppler study of ACA in the sagittal plane and ICA in coronal plane showed increased RI with bare loss of diastolic component $(\mathrm{g} \& \mathrm{~h})$, denoting increased ICT, and suggesting hypoxicischemic brain injury grade 4 according to wave shape and spectral analysis. 


\section{CASE (2)}

A newly born 6-days old full-term female baby delivered after normal unaided vaginal delivery for a multiparous woman and presented by disturbed level of consciousness. Images in coronal planes showed average hemispheric width $(26 \mathrm{~mm})$, average ventricular index $(9.8 \mathrm{~mm})$, average sino-cortical and cranio-cortical widths $(0.8 \mathrm{~mm} \& 1.6 \mathrm{~mm}$ respectively), and slit-like lateral ventricle with periventricular subependymal hemorrhagic spots. Doppler study in coronal plane showed mildly increased resistive index $(\mathrm{RI}=0.86)$ of ICA and in sagittal showed average resistive index ( RI $=0.8 \& 0.82$ respectively) of MCA and ACA with average diastolic components of both. Sonographic findings denoted increased ICT and subependymal periventricular hemorrhage (grade $1 \mathrm{ICH}$ ) and with Doppler study suggested grade 2 hypoxic-ischemic brain injuries (Fig. 3).
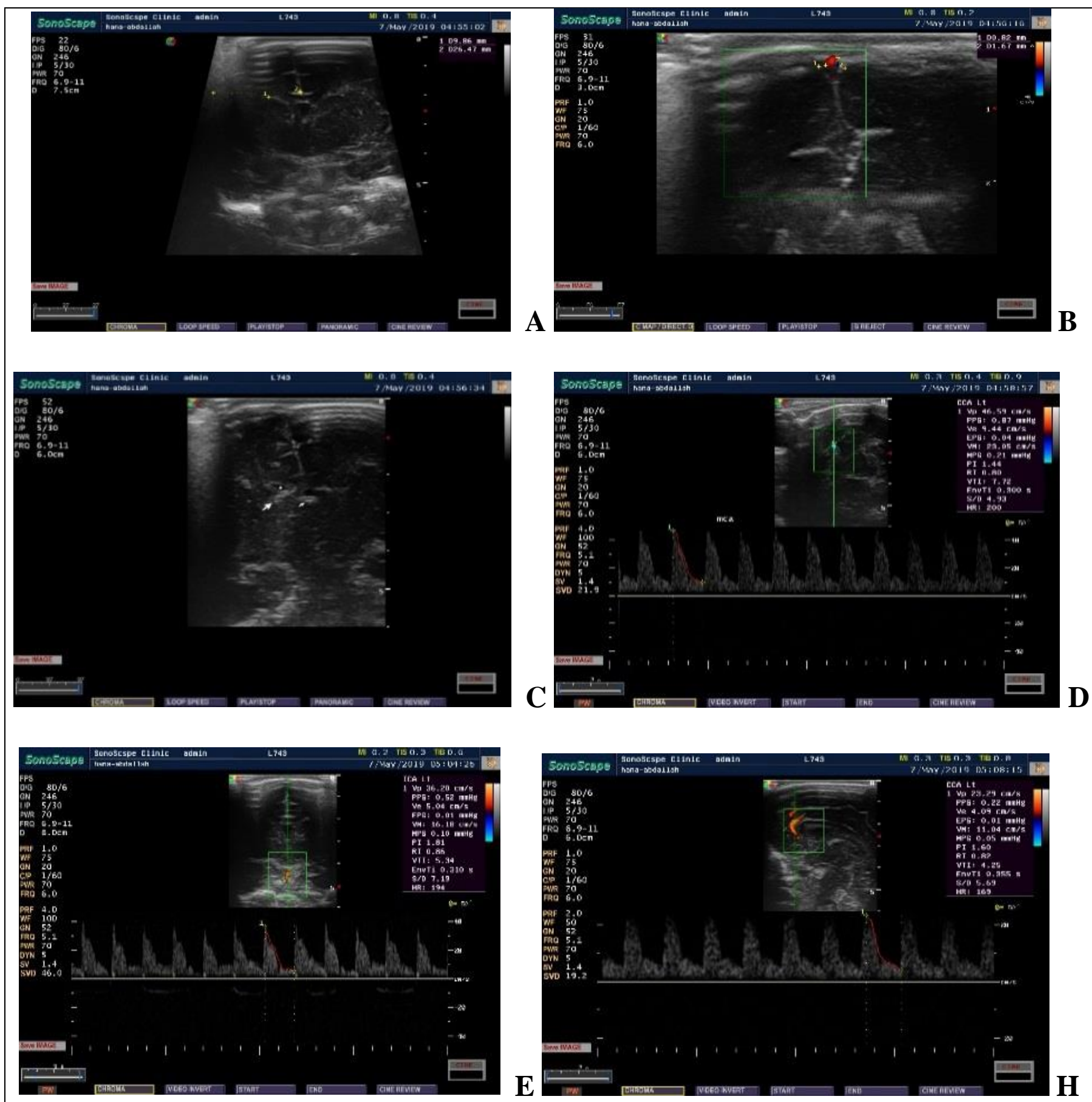

Fig. (3): GSUS imaging in coronal plane showed average biometric measurements, slit-like lateral ventricle (dot) \& periventricular subependymal hemorrhagic spots (arrows) (a-c). RI of MCA and ACA on Doppler study in sagittal plane was average ( $\mathrm{d} \& \mathrm{e}$ ), while Doppler study of ICA in coronal plane showed mildly increased RI; denoting increased ICT, and suggesting hypoxic-ischemic brain injury (grade 2) 


\section{CASE (3)}

A newly born 8-days old preterm male baby delivered after cesarean section for a multiparous woman and presented by repeated attacks of convulsions. Images in coronal planes showed dilated lateral ventricles $(21 \& 32 \mathrm{~mm})$ with internal turbid CSF fluid and peripherally located internal septations denoting blood clot formation and multiple periventricular cystic degenerations of white matter reflecting PVL grade 2-3. Images in coronal planes for the Doppler study of ICA showed increased RI (= 0.94) and barely loss of diastolic component. While, images in sagittal planes for the Doppler study of ACA showed decreased RI (=0.45) with an increased diastolic component. Overall sonographic findings included increased ICT and hemorrhagic hydrocephalus as well as PVL grade 2-3. Doppler findings suggested grade 3 hypoxic-ischemic brain injury (Fig. 4).
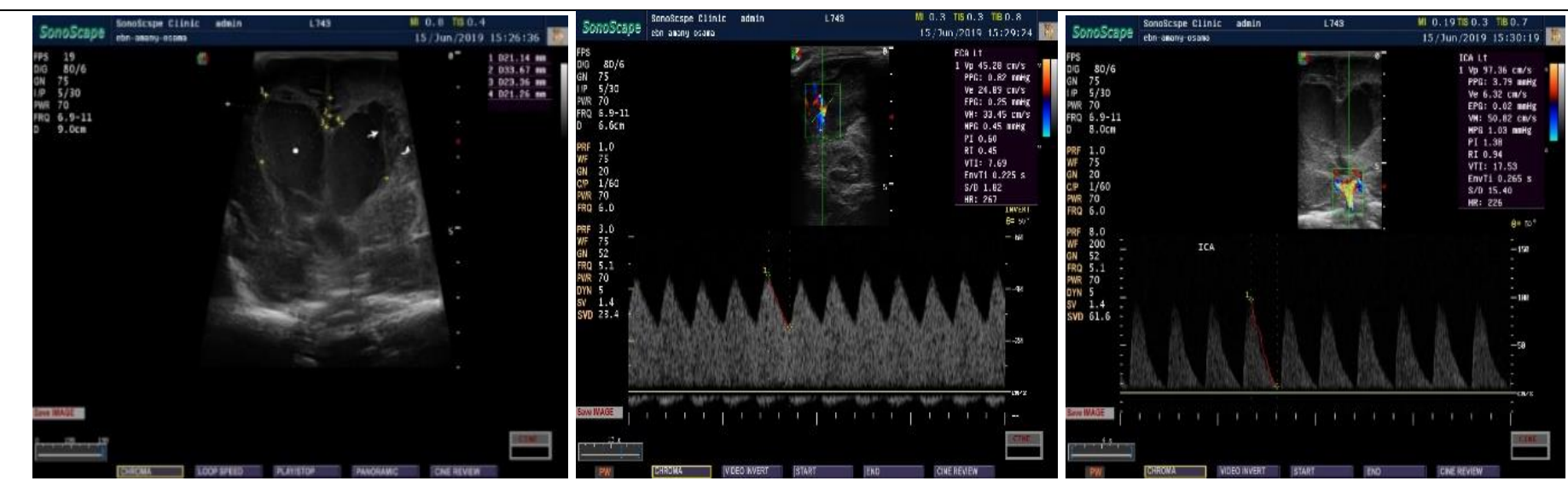

Fig. (4): GSUS in coronal plane showed dilated lateral ventricles with internal turbid CSF contents (dot) and peripherally located internal septations (arrow) indicating blood clot formation as well as multiple periventricular cystic degenerations of white matter reflecting PVL grade 2-3 (curved arrow A). Doppler study in sagittal plane showed decreased RI of ACA with increased diastolic component (B). While, Doppler study of ICA in sagittal plane showed increased RI $(=0.94)$ \& barely loss of diastolic component, suggesting increased ICT, indicating ischemic encephalopathy (grade 3).

Mortality was significantly correlated with Sarnat's clinical score, abnormality of RI of ACA and ICA, and presence and severity of HIE. While, handicapping of survivors showed a significant correlation with Sarnat's clinical score, the ventricular-hemisphere ratio on TC-GSUS and with an abnormality of RI of ACA and ICA (Table 5).

Table (5): Spearman's correlation between clinical scoring and US findings and outcome of the studied neonates

\begin{tabular}{|c|c|c|c|c|c|c|c|c|}
\hline \multirow{2}{*}{\multicolumn{2}{|c|}{$\begin{array}{l}\text { Parameters } \\
\text { Variables }\end{array}$}} & \multirow{2}{*}{$\begin{array}{l}\text { Sarnat's } \\
\text { score }\end{array}$} & \multirow{2}{*}{$\begin{array}{l}\text { Ventricular - } \\
\text { hemispheric } \\
\text { Ratio }\end{array}$} & \multicolumn{3}{|c|}{ RI of } & \multicolumn{2}{|c|}{ HIE } \\
\hline & & & & ACA & MCA & ICA & Presence & Severity \\
\hline \multirow{2}{*}{ Mortality } & Rho & 0.427 & 0.325 & 0.427 & 0.091 & 0.555 & 0.418 & 0.565 \\
\hline & $\mathrm{P}$ & 0.029 & 0.105 & 0.026 & 0.659 & 0.003 & 0.034 & 0.003 \\
\hline \multirow{2}{*}{ Handicapping } & Rho & 0.403 & -0.409 & -0.399 & 0.320 & 0.485 & 0.149 & 0.354 \\
\hline & $\mathrm{P}$ & 0.041 & 0.038 & 0.044 & 0.111 & 0.012 & 0.466 & 0.076 \\
\hline
\end{tabular}

Rho: Spearman's coefficient; RI: Resistive index; ACA: Anterior cerebral artery; MCA: Middle cerebral artery; ICA: Internal carotid artery; HIE: Hypoxic-ischemic encephalopathy.

ROC curve analysis of Sarnat's clinical scores and ultrasonographic findings as predictors for mortality as outcome defined abnormal RI of ACA and ICA and the severity of HIE as the significant predictors for mortality (Fig. 5). Regression analysis for the significant variables; the RI of ACA and ICA and the severity of HIE, defined the severity of HIE as the significant predictor of mortality as an outcome of the studied neonates with a standardized coefficient $(\beta)$ of 0.577 ( $\mathrm{p}=0.002$ ). Regarding prediction of handicapping, ROC curve analysis defined abnormal RI of ICA and ACA as the significant specific predictors for patients' handicapping (Fig. 6). Regression analysis assured the results of ROC analysis and defined abnormal RI of ICA as the most significant predictor for upcoming handicapping with a standardized coefficient of 0.535 ( $\mathrm{p}=0.005)$ (Table 6). 

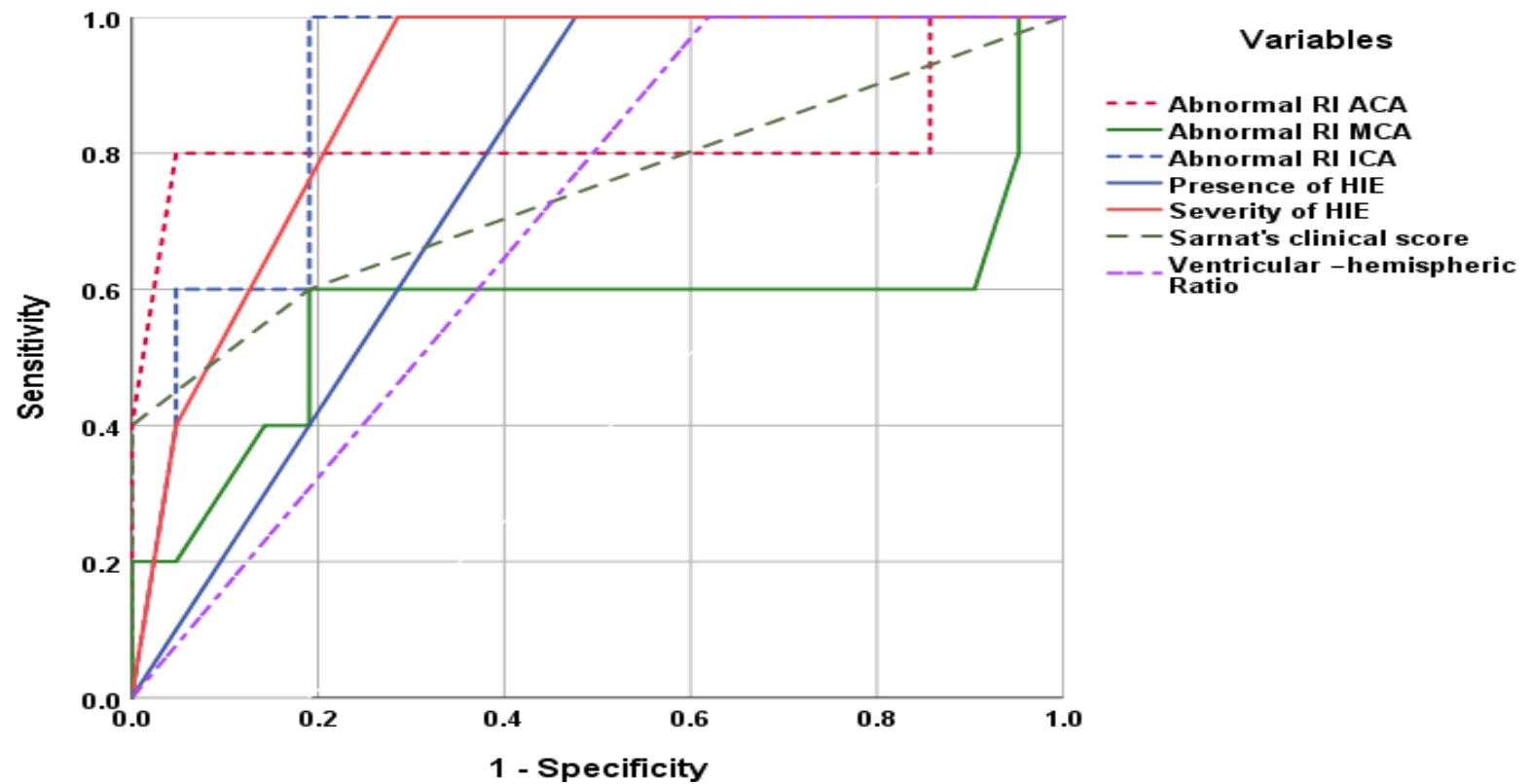

Fig. (5): ROC curve analysis of clinical scoring and US findings as predictors for upcoming mortality

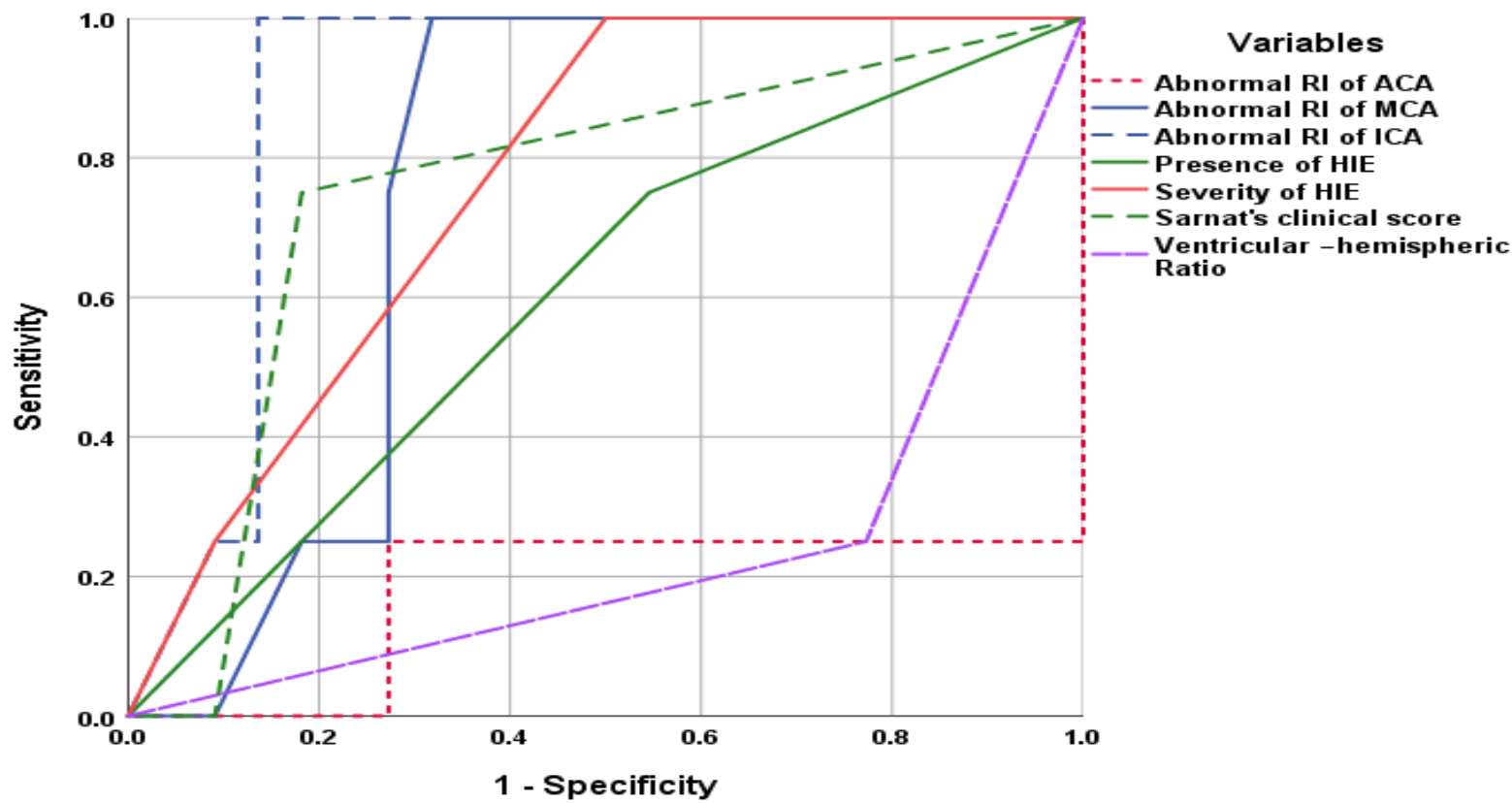

Fig. (6): ROC curve analysis of clinical scoring and US findings as predictors for upcoming handicapping.

Table (6): ROC curve analysis of clinical scoring and US findings as predictors for outcome of the studied neonates

\begin{tabular}{|c|c|c|c|c|c|c|}
\hline \multicolumn{3}{|c|}{ Outcome Variables Results } & AUC & SE & P-value & $95 \% \mathrm{CI}$ \\
\hline \multirow{7}{*}{ Mortality } & \multicolumn{2}{|c|}{ Sarnat's score } & 0.743 & 0.148 & 0.097 & $0.453-0.999$ \\
\hline & \multicolumn{2}{|c|}{ Ventricular -hemispheric Ratio } & 0.690 & 0.111 & 0.193 & $0.473-0.908$ \\
\hline & \multirow{3}{*}{ RI of } & ACA & 0.819 & 0.153 & 0.029 & 0.518-0.999 \\
\hline & & MCA & 0.567 & 0.192 & 0.649 & $0.191-0.942$ \\
\hline & & ICA & 0.905 & 0.061 & 0.006 & $0.785-0.999$ \\
\hline & \multirow{2}{*}{ HIE } & Presence & 0.762 & 0.096 & 0.074 & $0.574-0.950$ \\
\hline & & Severity & 0.890 & 0.065 & 0.008 & $0.763-0.999$ \\
\hline \multirow{7}{*}{ Handicapping } & \multicolumn{2}{|c|}{ Sarnat's score } & 0.750 & 0.133 & 0.118 & 0.489-0.999 \\
\hline & \multicolumn{2}{|c|}{ Ventricular-hemispheric Ratio } & 0.239 & 0.138 & 0.102 & $0.001-0.509$ \\
\hline & \multirow{3}{*}{ RI of } & $\mathrm{ACA}$ & 0.182 & 0.161 & 0.047 & $0.001-0.497$ \\
\hline & & MCA & 0.756 & 0.091 & 0.110 & $0.577-0.935$ \\
\hline & & ICA & 0.886 & 0.066 & 0.016 & $0.757-0.999$ \\
\hline & \multirow[t]{2}{*}{ HIE } & Presence & 0.602 & 0.150 & 0.522 & 0.309-0.896 \\
\hline & & \begin{tabular}{|l|} 
Severity \\
\end{tabular} & 0.767 & 0.103 & 0.095 & $0.566-0.968$ \\
\hline
\end{tabular}

RI: Resistive index; AUC: Area under the curve: SE: Standard error; CI: Confidence interval; ACA: Anterior cerebral artery; MCA: Middle cerebral artery; ICA: Internal carotid artery; HIE: Hypoxic-ischemic encephalopathy 
Considering the presence and severity of NHIE, as defined by Regression analysis is the significant predictor for mortality, paired-sample area difference under the ROC curves for RI levels of ACA, MCA and ICA could not differentiate which is the best predictor (Table 7). However, Regression analysis using the stepwise method defined high RI of ICA as the most specific predictor for the presence of HIE $(\beta=0.848, p<0.001)$, while high RI of ACA was the most sensitive predictor for severity $\operatorname{HIE}(\beta=0.762, \mathrm{p}<0.001)$.

Table (7): ROC curve analysis of RI of cranial arteries as judged by transcranial US Doppler as a predictor for presence and severity of HIE

\begin{tabular}{|c|c|c|c|c|}
\hline \multirow{4}{*}{$\begin{array}{l}\text { Presence } \\
\text { of HIE }\end{array}$} & & AUC (SE) & p & $95 \% \mathrm{CI}$ \\
\hline & $\mathrm{ACA}$ & $0.857(0.094)$ & 0.002 & $0.673-1.00$ \\
\hline & $\mathrm{MCA}$ & $0.839(0.101)$ & 0.003 & $0.642-1.00$ \\
\hline & ICA & $0.970(0.032)$ & $<0.001$ & $0.907-1.00$ \\
\hline \multirow{3}{*}{$\begin{array}{l}\text { Severity } \\
\text { of HIE }\end{array}$} & $\mathrm{ACA}$ & $0.143(0.094)$ & 0.002 & $0.00-0.327$ \\
\hline & MCA & $0.161(0.101)$ & 0.003 & $0.00-0.358$ \\
\hline & ICA & $0.030(0.032)$ & $<0.001$ & $0.00-0.093$ \\
\hline \multicolumn{2}{|c|}{ Paired-sample AUC difference } & AUC difference & $\mathrm{p}$ & $95 \% \mathrm{CI}$ \\
\hline \multicolumn{2}{|c|}{ ACA vs. MCA } & 0.018 & 0.262 & $\begin{array}{c}{[-0.013]-} \\
{[0.049]}\end{array}$ \\
\hline \multicolumn{2}{|c|}{ ACA vs. ICA } & -0.113 & 0.285 & $\begin{array}{c}{[-0.321]-} \\
{[0.094]}\end{array}$ \\
\hline \multicolumn{2}{|c|}{ MCA vs. ICA } & -0.131 & 0.246 & $\begin{array}{c}{[-0.352]-} \\
{[0.090]}\end{array}$ \\
\hline
\end{tabular}

RI: Resistive index; AUC: Area under the curve: SE: Standard error; CI: Confidence interval; HIE: Hypoxic-ischemic encephalopathy; ACA: Anterior cerebral artery; MCA: Middle cerebral artery; ICA: Internal carotid artery

\section{DISCUSSION}

The current study used Sarnat's clinical scoring for comparison versus US findings for its recently documented efficacy for prediction of the outcome of neonatal HIE, where Mrelashvili et al. ${ }^{[23]}$ and Morales et al. ${ }^{[24]}$ documented that total Sarnat's score of $>12$ indicated a high risk of death or moderate or severe disability, while a score of $<4$ indicated intact survival and was reassuring of a good outcome.

Application of TC-GSUS detected intracranial hemorrhage (ICH), brain edema, and/or hydrocephalus in $73.1 \%, 46.2 \%$, and $38.5 \%$ of studied neonates, respectively. These findings indicated increased cerebral blood flow resistance and suggested increased intracranial pressure and both may predispose or aggravate brain ischemia. Similarly, Bianchini et al. ${ }^{[25]}$ could identify ICH using B-mode US before the development of focal neurological signs or alterations in Doppler flow of MCA and optical nerve sheath diameter and suggested its use as a useful monitoring tool that could provide early clinical suspicion for the onset of ICH even before the development of intracranial hypertension or focal neurological deficits.

This study relied for discrimination between normal and abnormal RI on values previously identified by Zamora et al. ${ }^{[20]}$ who differentiated the normal range for RI according to gestational age (GA) as normal ranges for preterm and another for full-term neonates. In line with this decision, Elmfors et al. ${ }^{\text {[2] }}$ who showed the importance of considering GA for evaluating the RI and established normal values for RI of neonates who had GA ranging from 26 to 42 weeks.

Transcranial Doppler (TCD) detected abnormal RI of intracranial vessels, either separately or in combination, and assured the presence of increased cerebral blood flow resistance and ICP. These findings indicated the feasibility of the use of TCD as a noninvasive modality for evaluation of intracranial vessels and diagnosis of the presence of HIE and grading of its severity especially in cases of negative findings on TCGRUS. In line with these findings, multiple recent studies evaluated the diagnostic and prognostic validity of TCD in varied cases, where Cardim et al. ${ }^{[27]}$ demonstrated agreement between invasive and noninvasive ICP measurements using TCD and optic nerve sheet diameter ultrasound and suggested the potential role for a non-invasive technique for detection of $\mathrm{ICH}$. Thereafter, Sharawat $\boldsymbol{e t}$ al. ${ }^{[28]}$ assured the use of TCD as a non-invasive diagnostic modality for increased ICP and using Pearson correlation coefficient found the coefficients for optic nerve sheath diameter, pulsatility index, and resistive index were $0.98,0.914$, and 0.833 , respectively. Moreover, Roldán et al. ${ }^{[29]}$ conducted a meta-analysis for the most effective techniques for the non-invasive multimodal monitoring for traumatic brain injury and concluded that the near-infrared spectroscopy and TCD are the two most prominent and widely used technologies for non-invasive monitoring in TBI.

Statistical analyses of clinical scoring and US findings as predictors for the outcome; mortality and neurological affection, defined abnormal RI of ACA and ICA and the severity of HIE as the significant predictors for mortality, but the more severe the HIE, the more probability of mortality. Also, abnormal RI of ACA and ICA are the significant predictors for neurologic affection, but abnormal RI of ICA is the most significant. Moreover, differentially, Regression 
analysis defined abnormal RI of both ACA and ICA as highly diagnostic for worse outcomes. These findings support earlier reports that TCD could be used as a diagnostic and prognostic modality for infants with severe $\mathrm{HIE}{ }^{[31]}$ and can be used to guide medical therapy and management of cerebral hypoperfusion, which is associated with higher morbidity and mortality rates ${ }^{[31]}$. Moreover, Julkunen et al. ${ }^{[32]}$ found that TCD diagnosis of HIE Grade 2-3 and estimation of ACA-blood flow velocity can predict the outcome in asphyxiated infants better than cardiotocography, acid basement status, Apgar scores, or asphyxia markers. Thereafter, Lovett et al. ${ }^{[33]}$ documented favorable neurologic outcomes of patients who had TCD-detected near normal flow velocity with intermittently intact cerebral autoregulation. Canac et al. ${ }^{[34]}$ also documented that TCD appeared to be a valuable tool for assessing cerebral hemodynamics and could be used for accurate and reliable identification of cerebral blood flow velocity pulse onsets in neurocritical care settings.

Recently, Kumar et al. ${ }^{[35]}$ detected changes in early cerebral Doppler parameters that especially were significantly higher RI in patients with clinical features of neonatal encephalopathy in comparison with normal neonates. Doppler parameters are related with increasing severity of neonatal encephalopathy according to Sarnat's classification especially RI $<0.6$ and $>0.82$ that was associated with Doppler parameters with increasing severity according to Sarnat's classification.

\section{CONCLUSION}

TCD imaging of neonates with suspected HIE can diagnose HIE in neonates and abnormal RI of ACA and ICA might differentiate cases according to the severity of HIE that correlated with clinical severity scorings. Moreover, abnormal RI could predict the outcome of these patients with special regard to mortality and neurological handicapping. However, TC-GSUS could not predict the outcome or assure the diagnosis of HIE.

Financial support and sponsorship: Nil.

Conflict of interest: Nil.

\section{REFERENCES}

1. Cao Y, Liu H, Zhang J et al. (2020): Circular RNA cZNF292 silence alleviates OGD/R-induced injury through up-regulation of miR-22 in rat neural stem cells (NSCs). Artif Cells Nanomed Biotechnol., 48 (1): 594601.

2. Liljestrom L, Jonsson W (2018): Obstetric emergencies as antecedents to neonatal hypoxic ischemic encephalopathy, does parity matter? Acta Obstet Gynecol Scand., 97 (11): 1396-1404.

3. Rodriguez J, Li T, Xu Y et al. (2021): Role of apoptosis-inducing factor in perinatal hypoxic-ischemic brain injury. Neural Regen Res., 16 (2): 205-213.

4. Alkan H, Kahraman A, Mutlu A (2021): Early Spontaneous Movements of Infants with Hypoxic-
Ischemic Encephalopathy. Pediatr Phys Ther., 33 (1): 18-22.

5. Spagnoli C, Falsaperla R, Deolmi M et al. (2018): Symptomatic seizures in preterm newborns: a review on clinical features and prognosis. Ital J Pediatr., 44 (1): 115-119.

6. Di Stadio M, Gambacorta V, Giommetti G et al. (2019): Sensorineural Hearing Loss in Newborns Hospitalized in Neonatal Intensive Care Unit: An Observational Study. Int Tinnitus J., 23 (1): 31-36.

7. Charriaut-Marlangue C, Baud O (2018): A Model of Perinatal Ischemic Stroke in the Rat: 20 Years Already and What Lessons? Front Neurol., 9: 650-55.

8. Zhang L, Gao J, Zhao Y et al. (2021): The application of magnetic resonance imaging and diffusion-weighted imaging in the diagnosis of hypoxic-ischemic encephalopathy and kernicterus in premature infants Transl Pediatr., 10 (4): 958-966.

9. Aker K, Støen R, Eikenes L et al. (2020): Therapeutic hypothermia for neonatal hypoxic-ischaemic encephalopathy in India (THIN study): a randomised controlled trial. Arch Dis Child Fetal Neonatal Ed., 105 (4): 405-411.

10. Proisy M, Mitra S, Uria-Avellana C et al. (2016): Brain Perfusion Imaging in Neonates: An Overview. AJNR Am J Neuroradiol., 37 (10): 1766-1773.

11. Lindner $T$, Larsen $N$, Jansen $O$ et al. (2017): Selective arterial spin labeling in conjunction with phase-contrast acquisition for the simultaneous visualization of morphology, flow direction, and velocity of individual arteries in the cerebrovascular system. Magn Reson Med., 78 (4): 1469-1475.

12. Annink K, Meerts $L$, van der Aa $N$ et al. (2020): Cerebellar injury in term neonates with hypoxicischemic encephalopathy is underestimated. Pediatr Res., 20: 1-5.

13. van Leyen K, Klötzsch C, Harrer J (2011): Brain tumor imaging with transcranial sonography: state of the art and review of the literature. Ultraschall Med., 32 (6): 572-81.

14. La Rovere K, O'Brien N (2015): Transcranial Doppler Sonography in Pediatric Neurocritical Care: A Review of Clinical Applications and Case Illustrations in the Pediatric Intensive Care Unit. J Ultrasound Med., 34 (12): 2121-32.

15. Robba C, Taccone F (2019): How I use Transcranial Doppler. Crit Care, 23 (1): 420.

16. Sarnat H, Sarnat M (1976): Neonatal encephalopathy following fetal distress. A clinical and electroencephalographic study. Arch Neurol., 33: 696705.

17. Meijler G, Steggerda S (2019): Cranial Ultrasonography: Technical Aspects. In: Neonatal Cranial Ultrasonography. Cham, Switzerland, Springer, Pp: 7-21.

18. Meijler G, Steggerda S (2019): Part II ultrasound anatomy of the neonatal brain. In: Neonatal Cranial Ultrasonography. Cham, Switzerland, Springer, Pp: 266-366.

19. Guan B, Dai C, Zhang Y et al. (2017): Early diagnosis and outcome prediction of neonatal hypoxic-ischemic encephalopathy with color Doppler ultrasound. Diagnostic and Interventional Imaging, 98 (6): 469-75.

20. Zamora C, Tekes A, Alqahtani E et al. (2014): Variability of resistive indices in the anterior cerebral 
artery during fontanel compression in preterm and term neonates measured by transcranial duplex sonography. J Perinatol., 34 (4): 306-10.

21. Chalak L, Sánchez P, Adams-Huet B et al. (2014): Biomarkers for severity of neonatal hypoxic-ischemic encephalopathy and outcomes in newborns receiving hypothermia therapy. J Pediatr., 164(3):468-74.

22. Shellhaas $\mathrm{R}$, Thelen B, Bapuraj J et al. (2013): Limited short-term prognostic utility of cerebral NIRS during neonatal therapeutic hypothermia. Neurology, 81 (3): 249-55.

23. Mrelashvili A, Russ J, Ferriero D et al. (2020): The Sarnat score for neonatal encephalopathy: looking back and moving forward. Pediatr Res., 88 (6): 824-825.

24. Morales M, Montaldo P, Ivain $P$ et al. (2021): Association of Total Sarnat Score with brain injury and neurodevelopmental outcomes after neonatal encephalopathy. Arch Dis Child Fetal Neonatal Ed., 21: 1-5.

25. Bianchini A, D'Andrea R, Lepic B et al. (2019): Intracranial Hemorrhage Diagnosed with Transcranial Ultrasound in a Comatose, Postliver Transplant Patient. J Stroke Cerebrovasc Dis., 28 (11): 104357-63.

26. Elmfors A, Sandgren T, Ford K et al. (2019): Normal values of the resistivity index of the pericallosal artery with and without compression of the anterior fontanelle. Pediatr Radiol., 49 (5): 646-651.

27. Cardim D, Griesdale D, Ainslie P et al. (2019): A comparison of non-invasive versus invasive measures of intracranial pressure in hypoxic ischaemic brain injury after cardiac arrest. Resuscitation, 137: 221-228.

28. Sharawat I, Kasinathan A, Bansal A et al. (2020): Evaluation of Optic Nerve Sheath Diameter and
Transcranial Doppler As Noninvasive Tools to Detect Raised Intracranial Pressure in Children. Pediatr Crit Care Med., 20: 1-5.

29. Roldán M, Abay T, Kyriacou P (2020): Non-Invasive Techniques for Multimodal Monitoring in Traumatic Brain Injury: Systematic Review and Meta-Analysis. J Neurotrauma , 37 (23): 2445-2453.

30. Ilves P, Lintrop M, Talvik I et al. (2009): Low cerebral blood flow velocity and head circumference in infants with severe hypoxic ischemic encephalopathy and poor outcome. Acta Paediatr., 98 (3): 459-65.

31. Ducharme-Crevier L, Mills M, Mehta P et al. (2016): Use of Transcranial Doppler for Management of Central Nervous System Infections in Critically Ill Children. Pediatr Neurol., 65: 52-58.

32. Julkunen M, Uotila J, Eriksson $\mathrm{K}$ et al. (2012): Obstetric parameters and Doppler findings in cerebral circulation as predictors of 1-year neurodevelopmental outcome in asphyxiated infants. J Perinatol., 32 (8): 631-8.

33. Lovett M, Maa T, Chung M et al. (2018): Cerebral blood flow velocity and autoregulation in paediatric patients following a global hypoxic-ischaemic insult. Resuscitation, 126: 191-196.

34. Canac N, Ranjbaran M, O'Brien M et al. (2019): Algorithm for Reliable Detection of Pulse Onsets in Cerebral Blood Flow Velocity Signals. Front Neurol., 10: 1072-77.

35. Kumar I, Singh S, Kumar A et al. (2021): Early postnatal color Doppler changes in neonates receiving delivery room resuscitation with low 5 min Apgar scorea pilot study. J Perinatol., 41 (3): 486-493. 